\title{
Immobilized Metal Affinity Chromatography as a Drug Discovery Platform for
}

\section{Metalloenzyme Inhibitors}

Lukas Roth, Michael P. Gotsbacher, and Rachel Codd*

School of Medical Sciences (Pharmacology), The University of Sydney, New South Wales 2006, Australia

\section{Supporting Information}

Table of Contents

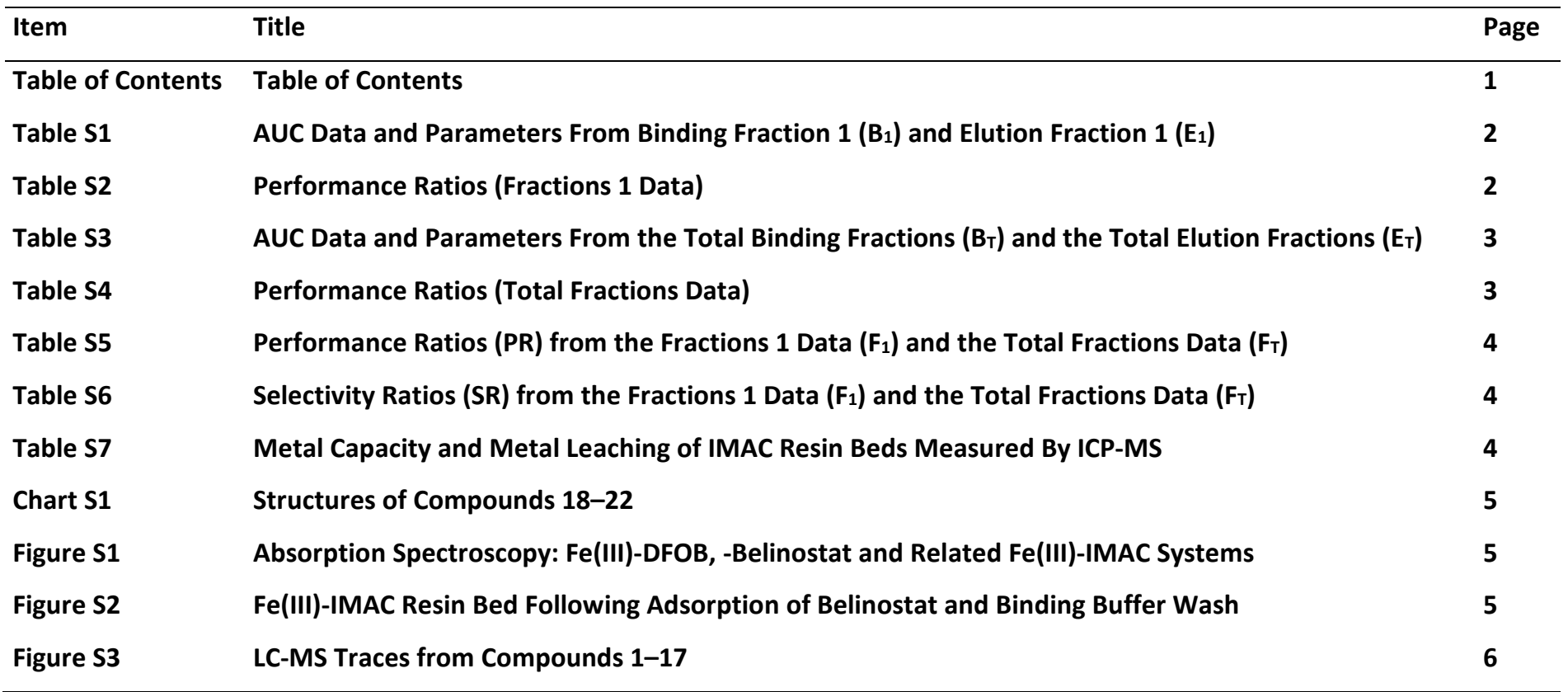


Table S1. AUC Data and Parameters From Binding Fraction $1\left(B_{1}\right)$ and Elution Fraction $1\left(E_{1}\right)$

\begin{tabular}{|c|c|c|c|c|c|c|c|c|c|c|c|c|c|c|c|c|c|c|c|c|c|}
\hline \multirow[t]{2}{*}{\begin{tabular}{|l|} 
No \\
\end{tabular}} & \multirow[t]{2}{*}{ Compound } & \multirow[t]{2}{*}{$\mathbf{B S D}^{a}$} & \multirow[t]{2}{*}{ ESD $^{b}$} & \multicolumn{6}{|c|}{$\mathrm{Cu}(\mathrm{II})$} & \multicolumn{6}{|c|}{$\mathrm{Fe}$ (III) } & \multicolumn{6}{|c|}{ Ga(III) } \\
\hline & & & & $B_{1}$ & $E_{1}$ & $T\left(B_{1}+E_{1}\right)$ & $\mathbf{A \mathbf { R } ^ { c }}$ & $\mathbf{R R}^{d}$ & $\mathbf{P R}^{e}$ & $B_{1}$ & $E_{1}$ & $\mathrm{~T}\left(\mathrm{~B}_{1}+\mathrm{E}_{1}\right)$ & $\mathbf{A \mathbf { R } ^ { c }}$ & $\mathbf{R R}^{d}$ & $\mathbf{P R}^{e}$ & $B_{1}$ & $E_{1}$ & $T\left(B_{1}+E_{1}\right)$ & $\mathbf{A R}^{c}$ & $\mathbf{R R}^{d}$ & $\mathbf{P R}^{e}$ \\
\hline 1 & lisinopril & 8857449 & 5524606 & 6258922 & 0 & 6258922 & 0.883285 & 0 & 0 & 6403242 & 0 & 6403242 & 0.903652 & 0 & 0 & 6601226 & 0 & 6601226 & 0.931592 & 0 & 0 \\
\hline 2 & enalaprilat & 18251136 & \begin{tabular}{|l|}
13158878 \\
\end{tabular} & 14500124 & 0 & 14500124 & 0.993097 & 0 & 0 & 14863342 & 0 & 14863342 & 1.017974 & 0 & 0 & 14657430 & 0 & 14657430 & 1.003871 & $\begin{array}{ll}0 \\
\end{array}$ & 0 \\
\hline 3 & captopril & 0 & 519006 & 0 & 0 & 0 & 0 & 0 & 0 & 0 & 0 & 0 & 0 & 0 & 0 & 54957.7 & 0 & 54957.7 & 0 & 0 & 0 \\
\hline 4 & omapatrilat & 0 & 148737 & 0 & 0 & 0 & 0 & 0 & 0 & 0 & 0 & 0 & 0 & 0 & 0 & 0 & 0 & 0 & 0 & 0 & 0 \\
\hline 5 & zofenopril & 3302947 & 3572269 & 3990979 & 0 & 3990979 & 1.510386 & 0 & 0 & 3848826 & 0 & 3848826 & 1.456588 & 0 & 0 & 3753271 & 37606.3 & 3790877 & 1.429198 & 0.00992 & 0.014178 \\
\hline 6 & entinostat & 22606036 & 16880998 & 10454074 & 224768.3 & 10678842 & 0.589153 & 0.021048 & 0.0124 & 17800534 & 79101.7 & 17879636 & 0.988185 & 0.004424 & 0.004372 & 17576430 & 150009.9 & 17726440 & 0.979293 & 0.008462 & 0.008287 \\
\hline 7 & tacedinaline & 7374612 & 5629169 & 5845124 & 24100.3 & 5869224 & 0.994319 & 0.004106 & 0.004083 & 5953329 & 19761.2 & 5973090 & 1.012017 & 0.003308 & 0.003348 & 5847869 & 23014.1 & 5870883 & 0.994623 & 0.00392 & 0.003899 \\
\hline 8 & panobinostat & 2698533 & 8377839 & 0 & 2472821 & 2472821 & 0.245968 & 1 & 0.245968 & 0 & 286782.2 & 286782.2 & 0.028526 & 1 & 0.028526 & 0 & 0 & 0 & 0 & \begin{tabular}{l|l}
0 \\
\end{tabular} & 0 \\
\hline 9 & vorinostat & 1509329 & 1188220 & 70627 & \begin{tabular}{|l|}
109741.3 \\
\end{tabular} & \begin{tabular}{|c|}
180368.3 \\
\end{tabular} & \begin{tabular}{|c|}
0.135457 \\
\end{tabular} & 0.608429 & 0.082416 & 0 & 0 & 0 & 0 & 0 & 0 & 0 & 587738.4 & \begin{tabular}{|l|}
587738.4 \\
\end{tabular} & 0.412198 & 1 & 0.412198 \\
\hline 10 & belinostat & 1637999 & 1124755 & 0 & 169420 & 169420 & 0.125524 & 1 & 0.125524 & 0 & 0 & 0 & 0 & 0 & 0 & 0 & 314992.4 & \begin{tabular}{|c|}
314992.4 \\
\end{tabular} & 0.233379 & 1 & 0.233379 \\
\hline 11 & 4-aminosalicylic acid & 2441406 & 1592367 & 1694144 & 0 & 1694144 & 0.867402 & 0 & 0 & 145401.5 & 152348.6 & 297750.1 & 0.154174 & 0.511666 & 0.078886 & 342553.8 & 990861 & 1333415 & 0.693935 & 0.7431 & 0.515663 \\
\hline 12 & zileuton & 1860189 & 1327347 & 924190.4 & 0 & 924190.4 & 0.621033 & 0 & 0 & 1348776 & 0 & 1348776 & 0.906344 & 0 & 0 & 1358189 & 0 & 1358189 & 0.912669 & 0 & 0 \\
\hline 13 & sulfasalazine & 5616268 & 4910492 & 1977610 & 3313378 & 5290988 & 1.002448 & 0.62623 & 0.627763 & 22369.2 & 4636182 & 4658551 & 0.79176 & 0.995198 & 0.787958 & 71786.6 & 4792502 & 4864289 & 0.829287 & 0.985242 & 0.817048 \\
\hline 14 & meclofenamic acid & 1869771 & 1512997 & 1571500 & 0 & 1571500 & 1.050596 & 0 & 0 & 1479499 & 0 & 1479499 & 0.989091 & 0 & 0 & 1511327 & 0 & 1511327 & 1.010369 & \begin{tabular}{l|l}
0 \\
\end{tabular} & 0 \\
\hline 15 & licofelone & 2276648 & 1982482 & 1870493 & 0 & 1870493 & 1.026999 & 0 & 0 & 1193845 & 748948 & 1942793 & 0.970303 & 0.385501 & 0.374053 & 1878708 & 115093.1 & 1993802 & 1.079889 & 0.057725 & 0.062337 \\
\hline 16 & tropolone & 1073227 & 150037 & 0 & 1621902 & 1621902 & 1.259365 & 1 & 1.259365 & 0 & 0 & 0 & 0 & 0 & 0 & 0 & 0 & 0 & 0 & 0 & 0 \\
\hline 17 & moxifloxacin & 22106822 & 13954649 & 223155.7 & 3978862 & 4202017 & 0.250225 & 0.946893 & 0.236936 & 955402.1 & 2536778 & 3492180 & 0.205511 & 0.726417 & 0.149287 & 2446779 & 399625.3 & 2846405 & 0.162214 & 0.140397 & 0.022774 \\
\hline
\end{tabular}

Table S1 Cont. AUC Data and Parameters From Binding Fraction $1\left(B_{1}\right)$ and Elution Fraction $1\left(E_{1}\right)$

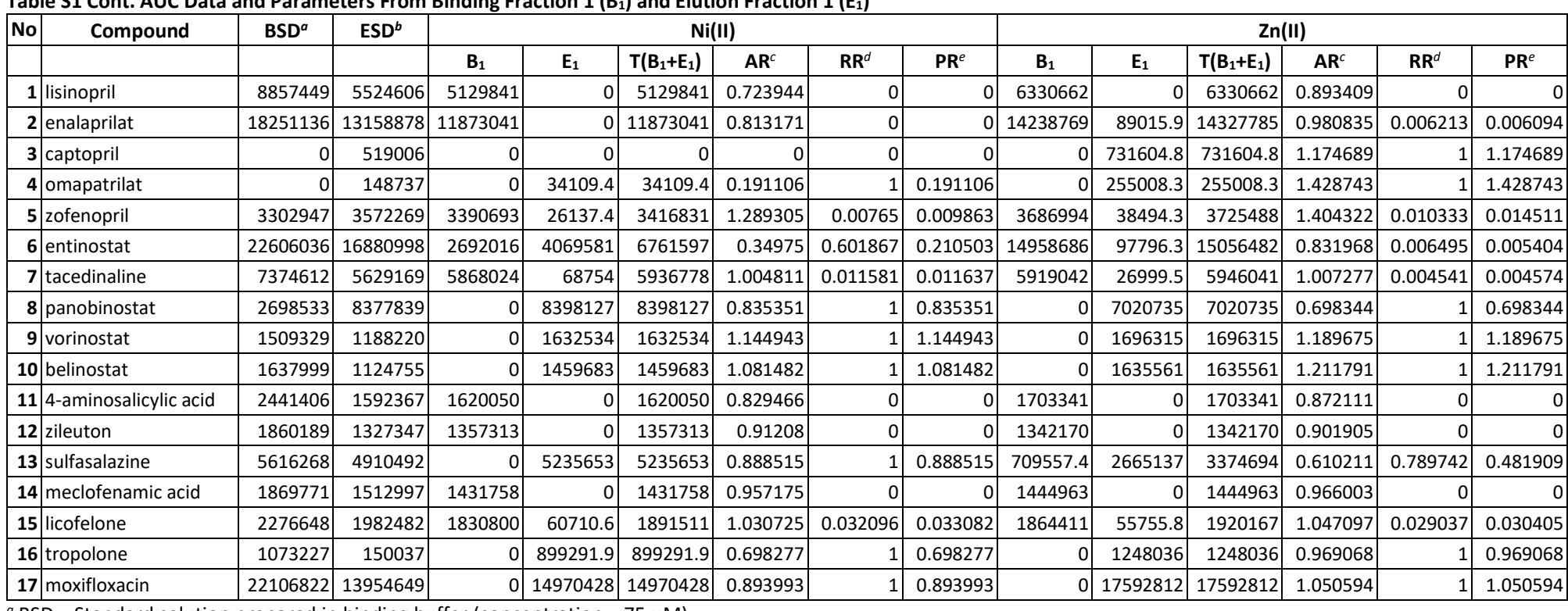

\begin{tabular}{l|l|l|}
1 & 0.893993 \\
\hline
\end{tabular} \begin{tabular}{l|l|l|l|l|}
0 & 17592812 & 17592812 & 1.050594 \\
\hline
\end{tabular}

Table S2. Performance Ratios (Fractions 1 Data)

\begin{tabular}{|c|c|c|c|c|}
\hline \multicolumn{5}{|c|}{ PR } \\
\hline $\mathrm{Cu}(\mathrm{II})$ & $\mathrm{Fe}(\mathrm{III})$ & $\mathrm{Ga}$ (III) & $\mathrm{Ni}(\mathrm{II})$ & $\mathrm{Zn}(\mathrm{II})$ \\
\hline 0 & 0 & 0 & 0 & 0 \\
\hline 0 & 0 & 0 & 0 & 0.01 \\
\hline 0 & 0 & 0 & 0 & 1.17 \\
\hline 0 & 0 & 0 & 0.19 & 1.43 \\
\hline 0 & 0 & 0.01 & 0.01 & 0.01 \\
\hline 0.01 & 0 & 0.01 & 0.21 & 0.01 \\
\hline 0 & 0 & 0 & 0.01 & 0 \\
\hline 0.25 & 0.03 & 0 & 0.84 & 0.70 \\
\hline 0.08 & 0 & 0.41 & 1.14 & 1.19 \\
\hline 0.13 & 0 & 0.23 & 1.08 & 1.21 \\
\hline 0 & 0.08 & 0.52 & 0 & 0 \\
\hline 0 & 0 & 0 & 0 & 0 \\
\hline 0.63 & 0.79 & 0.82 & 0.89 & 0.48 \\
\hline 0 & 0 & 0 & 0 & 0 \\
\hline 0 & \begin{tabular}{|l|}
0.37 \\
\end{tabular} & 0.06 & 0.03 & 0.03 \\
\hline 1.26 & 0 & 0 & 0.70 & 0.97 \\
\hline 0.24 & 0.15 & 0.02 & 0.89 & 1.05 \\
\hline
\end{tabular}

${ }^{b} \mathrm{ESD}=$ Standard solution prepared in elution buffer (concentration $=50 \mu \mathrm{M}$ ).

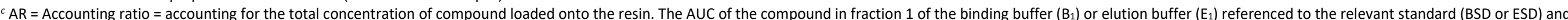

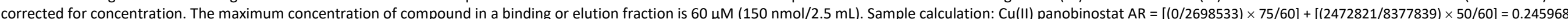

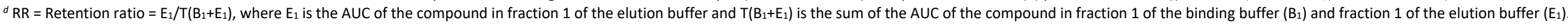

e $\mathrm{PR}$ - Performance ratio $=\mathrm{AR} \times \mathrm{RR}$. 
Table S3. AUC Data and Parameters From the Total Binding Fractions $\left(B_{T}\right)$ and the Total Elution Fractions $\left(E_{T}\right)$

\begin{tabular}{|c|c|c|c|c|c|c|c|c|c|c|c|c|c|c|c|c|c|c|c|c|c|}
\hline No & Compound & $\mathrm{BSD}^{a}$ & $\mathrm{ESD}^{b}$ & $\mathrm{Cu}(\mathrm{II})$ & & & & & & $\mathrm{Fe}(\mathrm{III})$ & & & & & & Ga(III) & & & & & \\
\hline & & & & $\mathbf{B}_{\mathbf{T}}$ & $\mathrm{E}_{\mathrm{T}}$ & $\mathrm{T}\left(\mathrm{B}_{\mathrm{T}}+\mathrm{E}_{\mathrm{T}}\right)$ & $\mathbf{A R}^{c}$ & $\mathbf{R R}^{d}$ & $\begin{array}{l}\mathbf{P R}^{e} \\
\end{array}$ & $\mathbf{B}_{\mathbf{T}}$ & $\mathrm{E}_{\mathrm{T}}$ & $\mathrm{T}\left(\mathrm{B}_{\mathrm{T}}+\mathrm{E}_{\mathrm{T}}\right)$ & $\mathbf{A R}^{c}$ & $\mathbf{R R}^{d}$ & $\begin{array}{l}\mathbf{P R}^{e} \\
\end{array}$ & $\mathrm{~B}_{\mathrm{T}}$ & $E_{\mathrm{T}}$ & $\mathrm{T}\left(\mathrm{B}_{\mathrm{T}}+\mathrm{E}_{\mathrm{T}}\right)$ & $\mathbf{A R}^{c}$ & $\mathbf{R R}^{d}$ & $\begin{array}{l}\mathbf{P R}^{e} \\
\end{array}$ \\
\hline 1 & lisinopril & 8857449 & 5524606 & 6314757 & 0 & 6314757 & 0.891165 & 0 & 0 & 6571077 & 0 & 6571077 & 0.927338 & 0 & 0 & 6688203 & 0 & 6688203 & 0.943867 & 0 & 0 \\
\hline 2 & enalaprilat & 18251136 & 13158878 & 14722091 & 0 & \begin{tabular}{|l|}
14722091 \\
\end{tabular} & 1.0083 & 0 & 0 & 15334688 & 0 & \begin{tabular}{|l|}
15334688 \\
\end{tabular} & 1.050256 & 0 & 0 & 15041970 & 0 & 15041970 & 1.030208 & 0 & 0 \\
\hline 3 & captopril & 0 & 519006 & 0 & 0 & 0 & 0 & 0 & 0 & 0 & 0 & 0 & 0 & 0 & 0 & 54957.7 & 0 & 54957.7 & 0 & 0 & 0 \\
\hline 4 & omapatrilat & 0 & 148737 & 0 & 0 & 0 & 0 & 0 & 0 & 0 & 0 & 0 & 0 & 0 & 0 & 0 & 0 & 0 & 0 & 0 & 0 \\
\hline 5 & zofenopril & 3302947 & 3572269 & 4122237 & 0 & 4122237 & 1.56006 & 0 & 0 & 4074543 & 0 & 4074543 & 1.54201 & 0 & 0 & 4029319 & 37606.3 & 4066925 & 1.533668 & 0.009247 & 0.014182 \\
\hline 6 & entinostat & 22606036 & 16880998 & 22647634 & 289239.3 & 22936873 & 1.266579 & 0.01261 & 0.015972 & 20455041 & 79101.7 & 20534143 & 1.134966 & 0.003852 & 0.004372 & 20618558 & 182078.9 & 20800637 & 1.149091 & 0.008754 & 0.010059 \\
\hline 7 & tacedinaline & 7374612 & 5629169 & 6312900 & 24100.3 & 6337000 & 1.073607 & 0.003803 & 0.004083 & 6426180 & 19761.2 & 6445941 & 1.092166 & 0.003066 & 0.003349 & 6644485 & 23014.1 & 6667499 & 1.12965 & 0.003452 & 0.0039 \\
\hline 8 & panobinostat & 2698533 & 8377839 & 41474 & 6650555 & 6692029 & 0.680734 & 0.993802 & 0.676515 & 47138 & 286782.2 & \begin{tabular}{|l|}
333920.2 \\
\end{tabular} & 0.050361 & 0.858835 & 0.043252 & 0 & 2281404 & 2281404 & 0.226928 & 1 & 0.226928 \\
\hline 9 & vorinostat & 1509329 & 1188220 & 579863 & 192460.3 & \begin{tabular}{|l}
772323.3 \\
\end{tabular} & 0.615211 & \begin{tabular}{|c|c|}
0.249197 \\
\end{tabular} & 0.153309 & 0 & 0 & 0 & 0 & 0 & 0 & 0 & 1031341 & 1031341 & 0.72331 & 1 & 0.72331 \\
\hline 10 & belinostat & 1637999 & 1124755 & 337908.3 & 272144 & \begin{tabular}{|l|}
610052.3 \\
\end{tabular} & 0.459499 & 0.446099 & 0.204982 & 0 & 106455 & 106455 & $\begin{array}{ll}0.078873 \\
\end{array}$ & 1 & \begin{tabular}{|c|}
0.078873 \\
\end{tabular} & 0 & 1384112 & 1384112 & 1.025492 & 1 & 1.025492 \\
\hline 11 & 4-aminosalicylic acid & 2441406 & 1592367 & 1744615 & 0 & 1744615 & 0.893243 & 0 & 0 & 2855738 & 152348.6 & 3008087 & 1.541867 & 0.050646 & 0.078089 & 2332354 & 1106220 & 3438574 & 1.773084 & 0.321709 & 0.570417 \\
\hline 12 & zileuton & 1860189 & 1327347 & 1211998 & 0 & 1211998 & 0.814433 & 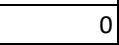 & 0 & 1726054 & 0 & 1726054 & 1.159865 & 0 & 0 & 1635676 & 0 & 1635676 & 1.099133 & 4 & 0 \\
\hline 13 & sulfasalazine & 5616268 & 4910492 & 5019434 & 3343357 & 8362791 & 1.684547 & 0.39979 & 0.673465 & 52742.9 & 6344108 & 6396851 & 1.088364 & 0.991755 & 1.07939 & 110971.5 & 6585857 & 6696829 & 1.142349 & 0.983429 & 1.123419 \\
\hline 14 & meclofenamic acid & 1869771 & 1512997 & 1904271 & 0 & 1904271 & 1.273064 & 0 & 0 & 1958112 & 0 & 1958112 & 1.309059 & 0 & 0 & 1855153 & 0 & 1855153 & 1.240228 & 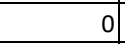 & 0 \\
\hline 15 & licofelone & 2276648 & 1982482 & 2100951 & 0 & 2100951 & 1.153533 & 0 & 0 & 2752120 & 748948 & 3501068 & 1.825878 & 0.21392 & 0.390592 & 2458942 & 115093.1 & 2574035 & 1.398468 & 0.044713 & 0.06253 \\
\hline 16 & tropolone & 1073227 & 150037 & 0 & 1621902 & 1621902 & 1.259365 & 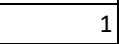 & 1.259365 & 0 & 0 & 0 & 0 & 0 & 0 & 0 & 0 & 0 & 0 & 4 & 0 \\
\hline 17 & moxifloxacin & 22106822 & 13954649 & 14990011 & 4091623 & 19081634 & 1.09193 & 0.214427 & 0.234139 & 12804875 & 2617920 & 15422794 & 0.880369 & 0.169744 & 0.149437 & 18308846 & 399625.3 & 18708472 & 1.059113 & 0.021361 & 0.022624 \\
\hline
\end{tabular}

Table S3 Cont. AUC Data and Parameters From the Total Binding Fractions $\left(\mathrm{B}_{\mathrm{T}}\right)$ and the Total Elution Fractions $\left(\mathrm{E}_{\mathrm{T}}\right)$

\begin{tabular}{|c|c|c|c|c|c|c|c|c|c|c|c|c|c|c|c|}
\hline \multirow[t]{2}{*}{ No } & Compound & $\mathrm{BSD}^{a}$ & ESD $^{b}$ & $\mathrm{Ni}(\mathrm{II})$ & & & & & & $\mathrm{Zn}(\mathrm{II})$ & & & & & \\
\hline & & & & $\mathbf{B}_{\mathbf{T}}$ & $E_{T}$ & $T\left(B_{T}+E_{T}\right)$ & $\mathbf{A R}^{c}$ & $\mathbf{R R}^{d}$ & $\mathbf{P R}^{e}$ & $B_{T}$ & $E_{T}$ & $T\left(B_{T}+E_{T}\right)$ & $\mathbf{A R ^ { c }}$ & $\mathbf{R R}^{d}$ & $\mathbf{P R}^{e}$ \\
\hline 1 & lisinopril & 8857449 & 5524606 & 6438046 & 0 & 6438046 & 0.908564 & 0 & 0 & 6412557 & 0 & 6412557 & \begin{tabular}{|l|}
0.904967 \\
\end{tabular} & 0 & \\
\hline 2 & enalaprilat & 18251136 & 13158878 & 16950624 & 0 & 16950624 & 1.160929 & 0 & 0 & 14614967 & 89015.9 & 14703983 & 1.0066 & 0.006054 & 0.006094 \\
\hline 4 & omapatrilat & 0 & 148737 & 0 & 34109.4 & 34109.4 & 0.191106 & 1 & 0.191106 & 0 & 255008.3 & 255008.3 & \begin{tabular}{|l|}
1.428743 \\
\end{tabular} & 1 & 1.428743 \\
\hline 5 & zofenopril & 3302947 & 3572269 & 3934174 & 26137.4 & 3960312 & 1.494985 & 0.0066 & 0.009867 & 3807084 & 38494.3 & 3845578 & 1.44977 & 0.01001 & 0.014512 \\
\hline 6 & entinostat & 22606036 & 16880998 & 21324030 & 4429170 & 25753200 & 1.397758 & 0.171985 & 0.240393 & 21422228 & 111688.3 & 21533917 & 1.190055 & 0.005187 & 0.006173 \\
\hline 9 & vorinostat & 1509329 & 1188220 & 0 & 1632534 & 1632534 & 1.144943 & 1 & 1.144943 & 0 & 1696315 & 1696315 & 1.189675 & 1 & 1.189675 \\
\hline 10 & belinostat & 1637999 & 1124755 & 0 & 1572641 & 1572641 & 1.165173 & 1 & 1.165173 & 0 & 1748865 & 1748865 & 1.295738 & 1 & 1.295738 \\
\hline 11 & 4-aminosalicylic acid & 2441406 & 1592367 & 1913409 & 0 & 1913409 & 0.979666 & 0 & 0 & 1742237 & 0 & 1742237 & \begin{tabular}{|l|}
0.892025 \\
\end{tabular} & 0 & 0 \\
\hline 12 & zileuton & 1860189 & 1327347 & 1749839 & 0 & 1749839 & 1.175848 & 0 & 0 & 1584774 & 0 & 1584774 & 1.064929 & 0 & 0 \\
\hline 13 & sulfasalazine & 5616268 & 4910492 & 129839.2 & 5477062 & 5606901 & 0.958381 & 0.976843 & 0.936188 & 5754219 & 2665137 & 8419356 & 1.73299 & 0.316549 & 0.548576 \\
\hline 14 & meclofenamic acid & 1869771 & 1512997 & 1963101 & 0 & 1963101 & 1.312394 & 0 & 0 & 1848573 & 0 & 1848573 & 1.235829 & 0 & 0 \\
\hline
\end{tabular}

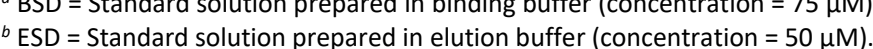

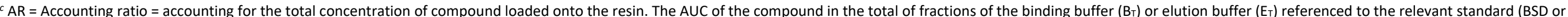

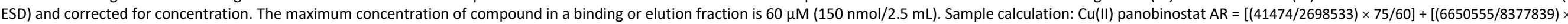
$50 / 60]=0.680734$

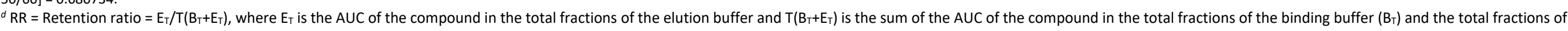
the elution buffer $\left(E_{T}\right)$.

${ }^{e} \mathrm{PR}-$ Performance ratio $=\mathrm{AR} \times \mathrm{RR}$.
Table S4. Performance Ratios (Total Fractions Data)

\begin{tabular}{|c|c|c|c|c|}
\hline \multicolumn{5}{|c|}{ PR } \\
\hline $\mathrm{Cu}(\mathrm{II})$ & $\mathrm{Fe}(\mathrm{III})$ & Ga(III) & $\mathrm{Ni}(\mathrm{II})$ & $\mathrm{Zn}(\mathrm{II})$ \\
\hline 0 & 0 & 0 & 0 & 0 \\
\hline 0 & 0 & 0 & 0 & 0.01 \\
\hline 0 & 0 & 0 & 0 & 1.17 \\
\hline 0 & 0 & 0 & 0.19 & 1.43 \\
\hline 0 & 0 & 0.01 & 0.01 & 0.01 \\
\hline 0.02 & 0 & 0.01 & 0.24 & 0.01 \\
\hline 0 & 0 & 0 & 0.01 & 0 \\
\hline 0.68 & 0.04 & 0.23 & 1.14 & 1.14 \\
\hline 0.15 & 0 & 0.72 & 1.14 & 1.19 \\
\hline 0.20 & 0.08 & 1.03 & 1.17 & 1.30 \\
\hline 0 & 0.08 & 0.57 & 0 & 0 \\
\hline 0 & 0 & 0 & 0 & 0 \\
\hline 0.67 & 1.08 & 1.12 & 0.94 & 0.55 \\
\hline 0 & 0 & 0 & 0 & 0 \\
\hline 0 & 0.39 & 0.06 & 0.03 & 0.03 \\
\hline 1.26 & 0 & 0 & 0.70 & 0.97 \\
\hline 0.23 & 0.15 & 0.02 & 1.12 & 1.08 \\
\hline
\end{tabular}


Table S5. Performance Ratios (PR) from the Fractions 1 Data $\left(F_{1}\right)$ and the Total Fractions Data $\left(F_{T}\right)$

\begin{tabular}{|c|c|c|c|c|c|c|c|c|c|c|c|}
\hline \multirow[t]{3}{*}{ No } & \multirow[t]{3}{*}{ Compound } & \multicolumn{10}{|c|}{ PR } \\
\hline & & \multicolumn{2}{|c|}{$\mathrm{Cu}(\mathrm{II})$} & \multicolumn{2}{|c|}{$\mathrm{Fe}(\mathrm{III})$} & \multicolumn{2}{|c|}{ Ga(III) } & \multicolumn{2}{|c|}{$\mathrm{Ni}(\mathrm{II})$} & \multicolumn{2}{|c|}{$\mathrm{Zn}(\mathrm{II})$} \\
\hline & & $F_{1}$ & $\mathrm{~F}_{\mathrm{T}}$ & $F_{1}$ & $\mathbf{F}_{\mathbf{T}}$ & $F_{1}$ & $\mathbf{F}_{\mathbf{T}}$ & $F_{1}$ & $\mathbf{F}_{\mathbf{T}}$ & $F_{1}$ & $\mathbf{F}_{\mathrm{T}}$ \\
\hline 2 & enalaprilat & 0 & 0 & 0 & 0 & 0 & 0 & 0 & 0 & 0.01 & 0.01 \\
\hline 3 & captopril & 0 & 0 & 0 & 0 & 0 & 0 & 0 & 0 & 1.17 & 1.17 \\
\hline 6 & entinostat & 0.01 & 0.02 & 0 & 0 & 0.01 & 0.01 & 0.21 & 0.24 & 0.01 & 0.01 \\
\hline 7 & tacedinaline & 0 & 0 & 0 & 0 & 0 & 0 & 0.01 & 0.01 & 0 & 0 \\
\hline 8 & panobinostat & 0.25 & 0.68 & 0.03 & 0.04 & 0 & 0.23 & 0.84 & 1.14 & 0.70 & 1.14 \\
\hline 9 & vorinostat & 0.08 & 0.15 & 0 & 0 & 0.41 & 0.72 & 1.14 & 1.14 & 1.19 & 1.19 \\
\hline 13 & sulfasalazine & 0.63 & 0.67 & 0.79 & 1.08 & 0.82 & 1.12 & 0.89 & 0.94 & 0.48 & 0.55 \\
\hline 14 & meclofenamic acid & 0 & 0 & 0 & 0 & 0 & 0 & 0 & 0 & 0 & 0 \\
\hline 15 & licofelone & 0 & 0 & 0.37 & 0.39 & 0.06 & 0.06 & 0.03 & 0.03 & 0.03 & 0.03 \\
\hline 16 & tropolone & 1.26 & 1.26 & 0 & 0 & 0 & 0 & 0.70 & 0.70 & 0.97 & 0.97 \\
\hline 17 & moxifloxacin & 0.24 & 0.23 & 0.15 & 0.15 & 0.02 & 0.02 & 0.89 & 1.12 & 1.05 & 1.08 \\
\hline
\end{tabular}

Table S6. Selectivity Ratios (SR) from the Fractions 1 Data $\left(F_{1}\right)$ and the Total Fractions Data $\left(F_{T}\right)$

\begin{tabular}{|c|c|c|c|c|c|c|c|c|c|c|c|}
\hline \multirow[t]{3}{*}{ No } & \multirow[t]{3}{*}{ Compound } & \multicolumn{10}{|c|}{ SR } \\
\hline & & \multicolumn{2}{|c|}{$\mathrm{Cu}(\mathrm{II})$} & \multicolumn{2}{|c|}{$\mathrm{Fe}(\mathrm{III})$} & \multicolumn{2}{|c|}{ Ga(III) } & \multicolumn{2}{|c|}{$\mathrm{Ni}(\mathrm{II})$} & \multicolumn{2}{|c|}{$\mathrm{Zn}(\mathrm{II})$} \\
\hline & & $\mathbf{F}_{1}{ }^{a}$ & $\mathbf{F}^{b}$ & $F_{1}$ & $\mathbf{F}_{\mathbf{T}}$ & $F_{1}$ & $\mathbf{F}_{\mathrm{T}}$ & $F_{1}$ & $F_{T}$ & $F_{1}$ & $\mathbf{F}_{\mathrm{T}}$ \\
\hline 1 & lisinopril & 0 & 0 & 0 & 0 & 0 & 0 & 0 & 0 & 0 & 0 \\
\hline 2 & enalaprilat & 0 & 0 & 0 & 0 & \begin{tabular}{l|l}
0 \\
\end{tabular} & 0 & 0 & 0 & 0 & 0 \\
\hline 3 & captopril & 0 & 0 & 0 & 0 & 0 & 0 & 0 & 0 & 1.00 & 1.00 \\
\hline 4 & omapatrilat & 0 & 0 & 0 & 0 & 0 & 0 & 0.13 & 0.13 & 1.00 & 1.00 \\
\hline 5 & zofenopril & 0 & 0 & 0 & 0 & 0 & 0 & 0 & 0 & 0 & 0 \\
\hline 6 & entinostat & 0.05 & 0.08 & 0 & 0 & 0.05 & 0.04 & 1.00 & 1.00 & 0.05 & 0.04 \\
\hline 7 & tacedinaline & 0 & 0 & 0 & 0 & 0 & 0 & 0 & 0 & 0 & 0 \\
\hline 8 & panobinostat & 0.30 & 0.60 & 0.04 & 0.04 & 0 & 0.20 & 1.00 & 1.00 & 0.83 & 1.00 \\
\hline 9 & vorinostat & 0.07 & 0.13 & 0 & 0 & 0.34 & 0.61 & 0.96 & 0.96 & 1.00 & 1.00 \\
\hline 10 & belinostat & 0.11 & 0.15 & 0 & 0.06 & 0.19 & 0.79 & 0.89 & 0.90 & 1.00 & 1.00 \\
\hline 11 & 4-aminosalicylic acid & 0 & 0 & 0.15 & 0.14 & 1.00 & 1.00 & 0 & 0 & 0 & 0 \\
\hline 12 & zileuton & 0 & 0 & 0 & 0 & 0 & 0 & 0 & 0 & 0 & 0 \\
\hline 13 & sulfasalazine & 0.71 & 0.60 & 0.89 & 0.96 & 0.92 & 1.00 & 1.00 & 0.84 & 0.54 & 0.49 \\
\hline 14 & meclofenamic acid & 0 & 0 & 0 & 0 & 0 & 0 & 0 & 0 & 0 & 0 \\
\hline 15 & licofelone & 0 & 0 & 1.00 & 1.00 & 0.16 & 0.15 & 0.08 & 0.08 & 0.08 & 0.08 \\
\hline 16 & tropolone & 1.00 & 1.00 & 0 & 0 & 0 & 0 & 0.56 & 0.56 & 0.77 & 0.77 \\
\hline 17 & moxifloxacin & 0.23 & 0.21 & 0.14 & 0.13 & 0.02 & 0.02 & 0.85 & 1.00 & 1.00 & 0.96 \\
\hline
\end{tabular}

${ }^{a} \mathrm{SR} \mathrm{F}_{1}=\mathrm{PR} \mathrm{F}_{1} / \mathrm{F}_{1} \max [\mathrm{Cu}(\mathrm{II}), \mathrm{Fe}(\mathrm{III}), \mathrm{Ga}(\mathrm{III}), \mathrm{Ni}(\mathrm{II}), \mathrm{Zn}(\mathrm{II})]$, where PR $\mathrm{F}_{1} \geq 0.2$.

${ }^{b} S R F_{T}=P R F_{T} / F_{T} \max [C u(I I), F e(I I I), G a(I I I), N i(I I), Z n(I I)]$, where $P R F_{T} \geq 0.2$.

Table S7. Metal Loading Capacity of and Metal Leaching from IMAC Resin Beds Measured By Inductively Coupled Plasma-Mass Spectrometry (ICP-MS). \begin{tabular}{|l|l|l}
\hline Metal lon & Metal Concentration \\
\hline
\end{tabular}

\begin{tabular}{|c|c|c|c|c|c|c|c|c|c|c|}
\hline Metal Ion & \multicolumn{10}{|c|}{ Metal Concentration } \\
\hline & $\mu \mathrm{mol}$ & & & ol $\mathrm{mL}^{-1} \mathrm{r}$ & & & $\mu \mathrm{mol}$ & $\mu \mathrm{mol} \mathrm{mL}^{-1}$ resin & $\mu \mathrm{mol}$ & $\mu \mathrm{mol}$ \\
\hline & $\operatorname{Exp} 1$ & Exp 1 & Exp 1 & Exp 1 & Exp 1 & $\operatorname{Exp} 1$ & Exp 2 & Exp 2 & $\operatorname{Exp} 2$ & $\operatorname{Exp} 2$ \\
\hline & & & Blank & & Inhibitor & & & & & \\
\hline & Loaded & Bound & Binding & Elution & Binding & Elution & Loaded & Bound & Unbound & Difference \\
\hline $\mathrm{Cu}(\mathrm{II})$ & 53.35 & 13.41 & 0.20 & 0.03 & 0.13 & 0.08 & 53.35 & 15.65 & 32.46 & 5.24 \\
\hline $\mathrm{Fe}(\mathrm{III})$ & 49.54 & 10.17 & 0 & 0 & 0.02 & 0 & 49.54 & 10.87 & 23.02 & 15.65 \\
\hline $\mathrm{Ga}(I I I)$ & 56.23 & 0.90 & 0.32 & 0.20 & 0.60 & 0.13 & 56.23 & 1.59 & 7.49 & 47.15 \\
\hline $\mathrm{Ni}(I I)$ & 52.55 & 13.75 & 0.01 & 0.02 & 0 & 0 & 52.55 & 14.99 & 31.08 & 6.47 \\
\hline $\mathrm{Zn}(\mathrm{II})$ & 51.33 & 12.96 & 0.42 & 0 & 0 & 0.15 & 56.93 & 16.50 & 40.42 & 0.01 \\
\hline
\end{tabular}

${ }^{a}$ Metal ion capacity on manufacturer's certificate of analysis: $12-18 \mu \mathrm{mol} \mathrm{mL}^{-1}$ resin. 
<smiles>Nc1ccc(-c2ccccc2)cc1NC(=O)c1ccccc1</smiles>

18<smiles>O=C(O)c1ccc(O)c(O)c1</smiles>

19<smiles>CC(=O)Nc1nnc(S(N)(=O)=O)s1</smiles>

20<smiles>Cc1cc(Cl)cc(Cn2cncc2C[C@H](N[C@@H](CC(C)C)C(=O)O)C(=O)O)c1</smiles>

21<smiles>CC(=O)NO</smiles>

22

Chart S1. Structures of 18-22 used in the in silico part of the study.

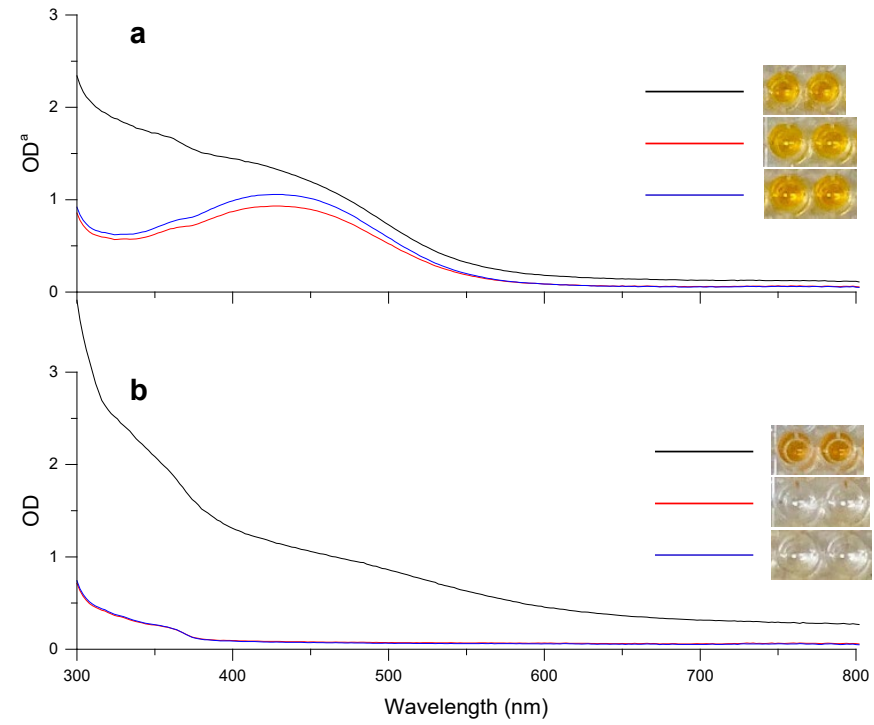

Figure S1. Absorbance profiles ( ${ }^{a}$ optical density corrected for 200- $\mu \mathrm{m}$ path length) from a standard solution (black) of a 1-mM complex of Fe(III) and (a) desferrioxamine B (DFOB) or (b) belinostat; or from the first (red) or second (blue) binding fractions $(2.5 \mathrm{~mL}$ ) from an $\mathrm{Fe}(\mathrm{III})-\mathrm{IMAC}$ column loaded with $2 \mu \mathrm{mol}$ of (a) DFOB or (b) belinostat.

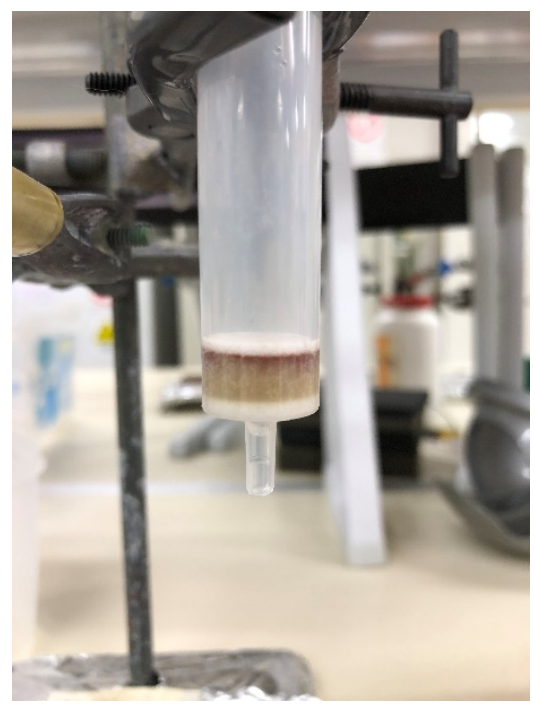

Figure S2. Fe(III)-IMAC resin following the adsorption of belinostat and washing the resin with one aliquot $(2.5 \mathrm{~mL})$ of binding buffer. 

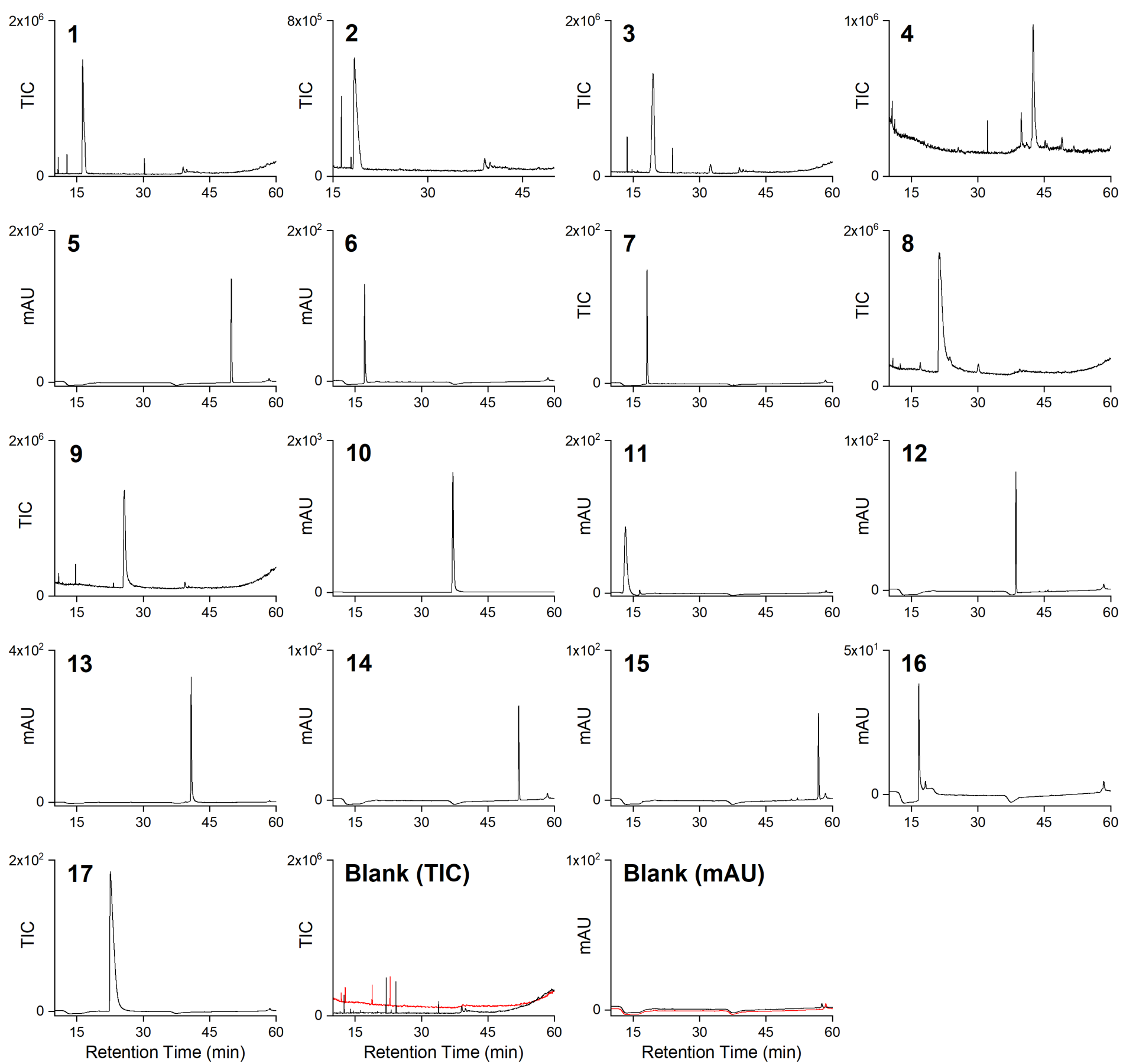

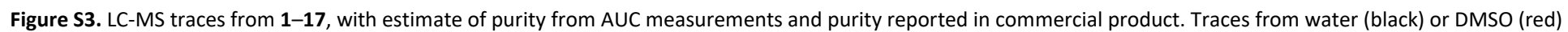
are shown as detected using TIC or absorbance.

\begin{tabular}{|l|l|l|l|}
\hline No & Compound & $\begin{array}{l}\text { Purity (\%) } \\
\text { Experimental }\end{array}$ & $\begin{array}{l}\text { Purity (\%) } \\
\text { Supplier Certificate }\end{array}$ \\
\hline $\mathbf{1}$ & lisinopril & 92 & 98 \\
\hline $\mathbf{2}$ & enalaprilat & 94 & 99.7 \\
\hline $\mathbf{3}$ & captopril & $99.9^{a}$ & 99.6 \\
\hline $\mathbf{4}$ & omapatrilat & $87^{b}$ & 100 \\
\hline $\mathbf{5}$ & zofenopril & 98 & 98 \\
\hline $\mathbf{6}$ & entinostat & 99.9 & 98 \\
\hline $\mathbf{7}$ & tacedinaline & 99 & 99.3 \\
\hline $\mathbf{8}$ & panobinostat & 96 & 95 \\
\hline $\mathbf{9}$ & vorinostat & 96 & 100 \\
\hline
\end{tabular}

\begin{tabular}{|l|l|l|l|}
\hline No & Compound & $\begin{array}{l}\text { Purity (\%) } \\
\text { Experimental }\end{array}$ & $\begin{array}{l}\text { Purity (\%) } \\
\text { Supplier Certificate }\end{array}$ \\
\hline $\mathbf{1 0}$ & belinostat & 99 & 97 \\
\hline $\mathbf{1 1}$ & 4-aminosalicylic acid & 96 & 98.9 \\
\hline $\mathbf{1 2}$ & zileuton & 98 & 98 \\
\hline $\mathbf{1 3}$ & sulfasalazine & 99.9 & 95 \\
\hline $\mathbf{1 4}$ & meclofenamic acid & 99.9 & 100 \\
\hline $\mathbf{1 5}$ & licofelone & 97 & 98 \\
\hline $\mathbf{1 6}$ & tropolone & 95 & 100 \\
\hline $\mathbf{1 7}$ & moxifloxacin & 99.9 & 100 \\
\hline & & & \\
\hline
\end{tabular}

${ }^{a}$ Includes $6 \%$ of captopril homodimer (3a).

${ }^{b}$ Includes $4 \%$ of omapatrilat homodimer (4a). 